\title{
OPTIMAL TRUSS DESIGN BASED ON AN ALGORITHM USING OPTIMALITY CRITERIA
}

\author{
JOHN E. TAYLOR \\ Department of Engineering Science and Applied Mechanies, University of Michigan, Ann Arbor, M1 48104, \\ U.S.A. \\ and \\ MArK P. Rossow \\ Department of Civil Engineering, Washington University, St. Louis, MO 63130, U.S.A.
}

(Received 30 August 1976; revised 10 March 1977)

\begin{abstract}
Abatract-A computational scheme is presented for the calculation of the optimal design of trusses. Constraints on the design variables (the cross-sectional areas) are considered. Linearly elastic behavior is assumed, and optimality criteria are derived, based on strain energy considerations. As in mathematical programming techniques, the optimum is approached through a sequence of designs, each differing slightly from its predecessor. The design changes to be made at each stage of the procedure are determined by application of the optimality criteria. The formulation is sufficiently general to permit the solution of the problem of predicting both optimal member size and member layout-given the loads and the location of the joints. The procedure is illustrated with a number of numerical examples.
\end{abstract}

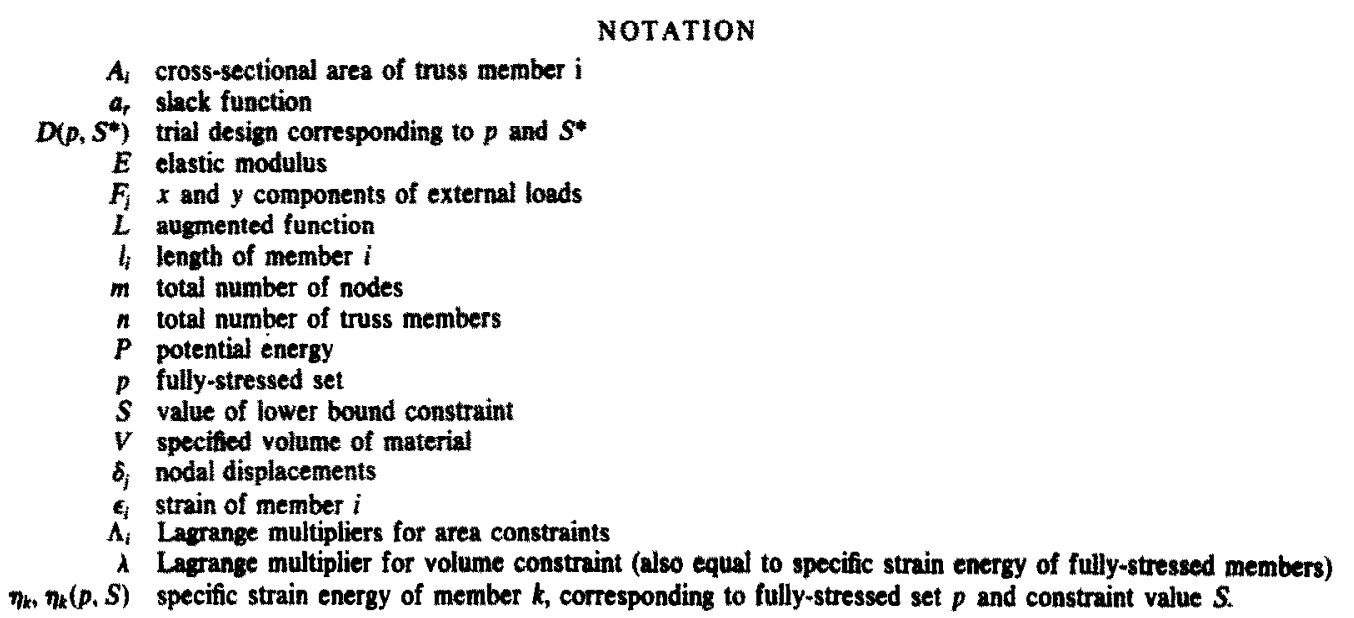

\section{INTRODUCTION}

Mathematical programming techniques and the use of optimality criteria are probably the two most widely-used solution techniques in the field of optimal structural design. Although both approaches have their advantages and many diverse applications of each have appeared in the technical literature, both also have certain drawbacks. For example, applications of optimality criteria tend to be limited to problems with relatively few design constraints, and even these few constraints must be rather simple if the mathematical form of the optimality criteria is to be tractable. Mathematical programming techniques, on the other hand, can treat problems with constraints which are both more numerous and more complicated. Unfortunately, however, the algorithms employed to search for the optimum tend to consume considerable computer time as the number of design variables in a problem is increased.

In the present paper, an algorithm is given which resembles a technique of mathematical programming in that it proceeds by stages, with an improved design generated at each stage. However, in contrast to most mathematical programming methods, the improved design is 
identified at each stage by the application of optimality criteria. The algorithm is explained and illustrated by application to the optimal design of a truss, where member cross-sectional areas are taken as the design variables. Dorn et al.[1] have treated a similar problem by linear programming.

A description of the algorithm has been given previously [2], and for completeness that description will be given here as well, supplemented by additional explanation at several key points. In general, the present work constitutes a more complete and detailed account of the theory described in [2], and in addition a number of significant examples are given here.

\section{ENERGY FORMULATION}

Consider the problem of finding the maximum stiffness design of a planar truss, given a specified total volume of material to be allocated to the various members of the truss, and specifying inequality constraints on the truss members cross-sectional areas. The connectivity of the truss is unrestricted; however, locations of nodes are specified beforehand, and the possibility of member buckling is ignored. Taylor [3] and Hiley [4] have shown how a problem of the type just described may be formulated by the use of the potential energy function of the structure. In the present paper a similar energy formulation will be used. The potential energy of the truss may be written

$$
P=\sum_{i=1}^{n} l_{1} A \eta_{i}-\sum_{i=1}^{2 m} F_{j} \delta_{j}
$$

where

$n=$ total number of truss members assuming each node connected to all other nodes by a member,

$m=$ total number of nodes of truss,

$A_{i}=$ cross-sectional area of member $i$

$l_{i}=$ length of member $i$,

$F_{l}=x$ and $y$ components of external loads applied at interior nodes and numbered consecutively,

$\delta_{i}=$ nodal displacements, numbered corresponding to $F_{j}$;

and $\eta_{i}$ represents the specific strain energy, defined by

$$
\eta_{i}=E \epsilon_{i}^{2} / 2
$$

where $E$ is the elastic modulus and $\epsilon_{i}$ is the strain of member $i$ (a linear function of nodal displacements). The volume constraint is

$$
\sum_{i=1}^{n} A_{i} d_{i}=V
$$

where $V$ is the specified volume of material. The inequality constraints are

$$
A_{i} \geq S
$$

where $S$ is the specified lower bound constraint.

It can be shown that the problem of maximum stifiness design is equivalent to that of maximizing the potential energy $P[3,5]$.

The constraints may be introduced directly into the problem formulation by defining the slack functions $a_{r}$ by

$$
A_{r}-a_{r}^{2}=S, \quad r=1,2 \ldots, n
$$

and introducing Lagrange multipliers $\lambda$ and $\Lambda_{i}$ to form the augmented function

$$
L=P+\lambda\left(V-\sum_{i=1}^{n} A_{i} l_{i}\right)+\sum_{i=1}^{n} \Lambda_{i}\left(S-A_{i}+a_{i}^{2}\right)
$$


Requiring the first derivatives of $L$ with respect to $\delta_{k}, A_{r}$, and $a_{r}$ to vanish gives

$$
\begin{gathered}
\sum_{i=1}^{n} l_{i} A_{i} \frac{\partial \eta_{i}}{\partial \delta_{k}}-F_{k}=0 \\
\eta l_{r}-\lambda l_{r}-\Lambda_{r}=0 \\
\Lambda_{r} a_{r}=0 .
\end{gathered}
$$

while application of the Kuhn-Tucker theorem of non-linear programming gives

$$
\Lambda, \leq 0 \text {. }
$$

These equations can be shown to be both necessary and sufficient for optimality $[3,6,7]$. A basic assumption about the optimal design problem formulated above will now be made.

It is assumed that for every value of $S$ in the interval $0<S \leq V /\left(\sum_{i=1}^{n} l_{i}\right)$ an optimal design exists. That is, the optimal design is assumed to be a function of $S$. Furthermore, this function is assumed continuous.

It is of interest to note that at least one optimal design can always be found easily for the value of the lower bound constraint given by

$$
S=V /\left(\sum_{i=1}^{n} l_{i}\right)
$$

For by eqn (4) all admissible designs must satisfy

$$
A_{j} \geq S^{*} \geq V /\left(\sum_{i=1}^{n} l_{i}\right), \quad j=1,2, \ldots, n .
$$

However the strict inequality in eqn (12) cannot apply for any $j$ since this would violate the volume constraint in eqn (3). Thus the optimal design for the value of $S$ in eqn (11) must be the "equally-sized" design

$$
A_{j}=V /\left(\sum_{i=1}^{n} l_{i}\right), \quad j=1,2, \ldots, n
$$

\section{OBSERVATIONS ON THE GOVERNING EQUATIONS}

Inspection of the preceding set of governing eqns (3)-(10) leads to several observations of later use in this paper. First note that when a member area $A_{r}$ in the optimal design is strictly greater than the lower bound constraint value $S$, then the corresponding slack function $a_{r} \neq 0$ by eqn (5) and $\Lambda_{r}=0$ by eqn (9), but then eqn (8) yields

$$
\eta_{r}=\lambda
$$

Thus all members with areas greater than $S$ are stressed to the same level.

Note that by eqn (2), eqn (13) may be written as a linear equation in the strain $\epsilon_{r}$ and hence linear in the nodal displacements:

$$
\epsilon_{\mathrm{r}}= \pm \sqrt{2 \lambda / E}
$$

Next consider a member $t$ in the optimal design which is stressed below the level $\lambda$ (eqns (8) and (10) exclude the possibility that an element in the optimal design is stressed above the level ג.): 
Then by eqn (8) $\Lambda_{t} \neq 0$ and so eqns (9) and (5) imply

$$
A_{t}=S \text {. }
$$

The implication of eqns (14) and (16) may be summarized by saying that the members of the optimal design may be divided into two groups: fully-stressed members $\left(\eta_{r}=\lambda\right.$ and $\left.A_{r}>S\right)$ and members at the constraint $\left(\eta_{t}<\lambda\right.$ and $\left.A_{t}=S\right)$. As shall be discussed later, under certain conditions borderline cases exist where a member is both fully-stressed and at the constraint.

A second observation about the governing equations for the optimal design problem can be made with the help of the fully-stressed condition, eqn (14). Introducing eqns (14) and (2) into the equilibrium relations (eqn 7 ) yields

$$
\sqrt{2 \lambda E} \sum_{r} e_{\lambda_{r}} A_{r} \frac{\partial \epsilon_{r}}{\partial \delta_{k}}+S \sum_{i} l_{l} \frac{\partial \eta_{t}}{\partial \delta_{k}}-F_{k}=0
$$

where the first summation is over the set of fully-stressed members, and the second summation over the set of members at the constraint (hence areas equal $S$ ). $e_{r}$ is the sign associated with member $r$ (compression or tension).

Equations (14) and (17) have been formulated for the problem of maximum stiffness design for a fixed volume of material $V$. The maximum specific strain energy $\lambda$ is found as part of the solution. However, this problem may be shown [8] to be equivalent to the problem of minimum volume design for specified $\lambda$. From now on in this paper it will be assumed that a value of $\lambda$ is specified. The solution corresponding to this value of $\lambda$ may later be made to correspond to some specified volume of material by multiplying all results by a common factor.

With $\lambda$ specified, eqns (14) and (17) become linear equations in the remaining unknowns $\delta_{k}$ and $A_{r}$. Thus once it has been determined which members are to be fully-stressed in the optimal design, the areas and nodal displacements may be calculated by solving a linear system of equations.

\section{FULLY.STRESSED SET AND TRIAL DESIGN}

The principle difficulty then in finding an optimal design lies in determining which members are fully-stressed. In view of this situation and for convenience in the discussion to follow, the following definitions will be made.

Suppose that a subset of the $n$ members of the truss have specific strain energy $\lambda$, as well as specified signs, and do not violate nodal displacement compatibility. These members will be called a "fully-stressed set".

Suppose that a fully-stressed set $p$ has been designated and a value of the lower bound constraint specified, $S=S^{*}$. In general, it is not known beforehand if $p$ corresponds to an optimal design for $S=S^{*}$. However, knowing $p$ and $S^{*}$, we can nevertheless determine a corresponding set of areas and displacements by writing eqns (17) and (14) for the fully-stressed set $p$ and then solving these equations.

The set of areas and displacements found in this way will be written $D\left(p, S^{*}\right)$ and will be called the "trial design corresponding to $p$ and $S^{*}$." Note that by assumption the trial design is a continuous function of the lower bound constraint, for a fixed $p$.

Once a trial design $D\left(p, S^{*}\right)$ has been calculated, eqns (10) and (4) may be used to determine if the trial design is also an optimal design. If $D\left(p, S^{*}\right)$ is optimal, then $p$ will be called the "optimal fully-stressed set corresponding to $S^{*}$."

\section{BASIS FOR ALGORITHM}

Using the definitions just introduced, we can now discuss the basis for an algorithm for finding the optimal design.

Starting with a fully-stressed set $r$ and a value of $S=S^{*}$ such that $D\left(r, S^{*}\right)$ is optimal (finding such a starting design presents no difficulties, as was observed earlier), $S$ is repeatedly reduced and $D(r, S)$ recalculated until a value of $S$ is found for which $D(r, S)$ is non-optimal. Since the cause of the non-optimality must lie in the incorrect choice of fully-stressed members, a method is needed for identifying those members which must be added to or deleted from the 
optimal fully-stressed set as $D$ decreases. Such a method may be derived from a close examination of the optimal designs in the neighborhood of a point where the optimal fullystressed set changes.

Consider the particular case where a single member, $j$ say, is to be added to the optimal fully-stressed set. In Fig. $1, S=S_{c}$ is the value of the lower bound constraint for which $\eta_{i}$ first equals the constraint value $\lambda$ as $S$ is decreased from a value $S_{2}$ slightly above $S_{\mathrm{c}}$ to a value $S_{1}$ slightly below $S_{c}$. Note that, for $S=S_{c}$, member $j$ is an example of a "borderline" case referred to earlier $\left(A_{j}=S_{c}\right.$ and $\left.\eta_{j}=\lambda\right)$. We specifically exclude from consideration the possibility that more than one borderline element exists at $S=S_{c}$. This restriction will be discussed later in this paper.

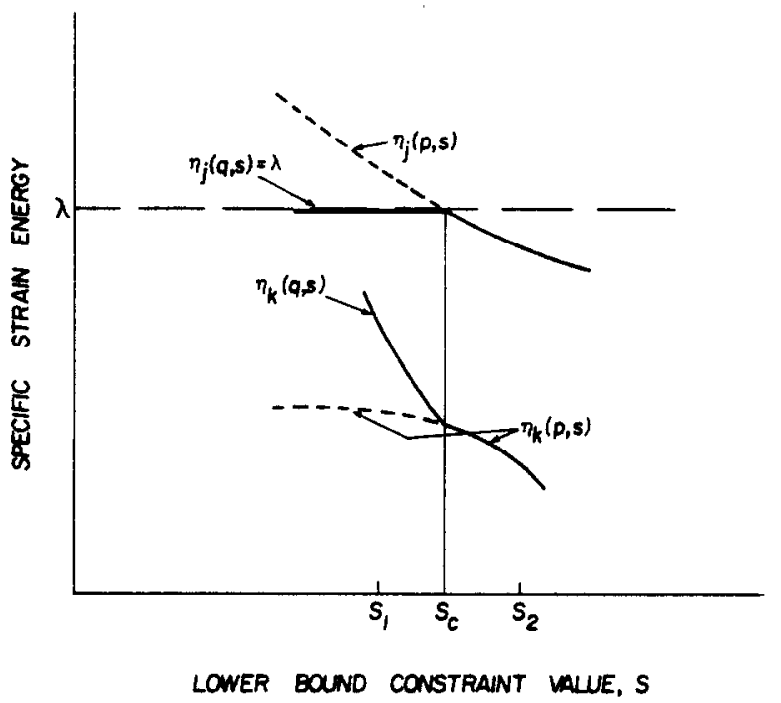

Fig. 1. Member $j$ to be added to optimal fully-stressed set.

If $p$ denotes the full-stressed set for which $D(p, S)$ is optimal for $S_{2} \geq S \geq S_{c}$, then $D(p, S)$ is non-optimal for $S_{c}>S \geq S_{1}$, since by hypothesis $p$ lacks the fully-stressed member $j$.

Denote by $q$ the fully-stressed set obtained from $p$ by adding member $j$ and consider a member, $k$ say, which belongs to neither $p$ nor $q$. By hypothesis,

$$
\eta_{k}\left(p, S_{c}\right)=\eta_{k}\left(q, S_{c}\right)<\lambda
$$

Furthermore since $\eta_{k}(p, S)$ and $\eta_{k}(q, S)$ are continuous functions of $S$, it follows that

$$
\eta_{k}(p, S)<\lambda \text { and } \eta_{k}(q, S)<\lambda
$$

for $S_{1} \leq S<S_{c}$. For the same range of $S$, it must also be true that

$$
\eta_{i}(p, S)>\lambda
$$

since $D(p, S)$ has been assumed to be non-optimal. Thus the member to be added to the fully-stressed set $p$ to form the optimal fully-stressed set $q$ (for $S_{1} \leq S<S_{c}$ ) may be determined by examining the non-optimal design $D\left(p, S_{1}\right)$-the member to be added is that member with specific strain energy exceeding $\lambda$. The sign associated with the member $j$ to be added is identical to the sign of member $j$ in $D\left(q, S_{1}\right)$, as may be established by a continuity argument similar to that given above.

The preceding discussion dealt with the procedure for identifying the member to be added to the optimal fully-stressed set as $S$ decreases. An analogous procedure can be developed for identifying the member to be deleted from the optimal fully-stressed set. Proceeding as in the previous paragraphs, it can be shown that the members of the optimal fully-stressed set can be identified by inspection of a non-optimal design $D\left(p, S_{1}\right)$-the criterion being that the member 
in $p$ whose area is less than $S_{1}$ is to be deleted from $p$ to form the optimal fully-stressed set.

The arguments of the preceding paragraphs depend in an essential way on knowledge of the approximate location of a point such as $S=S_{c}$ where a change occurs in the optimal fully-stressed set. This information can be provided through application of a modified version of an interval-halving algorithm such as the one given in Fig. 2. The algorithm starts with a fully-stressed set $p$ and two values of $S, S_{1}$ and $S_{2}$ say $\left(S_{1}<S_{2}\right)$, such that $D\left(p, S_{1}\right)$ is non-optimal and $D\left(p, S_{2}\right)$ is optimal. Then, for a specified number $\epsilon>0$, the algorithm produces two new values of $S, S_{3}$ and $S_{4}$ say $\left(S_{1} \leq S_{3}<S_{4} \leq S_{2}\right)$, such that $D\left(p, S_{3}\right)$ is non-optimal, $D(p$, $\left.S_{4}\right)$ is optimal, and

$$
S_{4}-S_{3}<\epsilon
$$

To summarize what has been accomplished thus far, we can say that a method has been developed for finding the optimal fully-stressed set for decreasing values of the lower bound constraint $S$. This method may be applied for decreasing $S$ until $S$ approaches some specified limit $S^{* *}$ or $S$ approaches zero-the layout problem. A flow chart of the entire optimal truss design algorithm showing the major computational blocks and logical branching is given in Fig. 3. Note that the fully-stressed set $p$ is modified one member at a time and checked for optimality after each modification. The value of $S$ is reduced only when the optimality conditions are satisfied.

A final remark on the algorithm should be added here. In developing the method for adding or deleting fully-stressed members, the assumption was made that only one element at a time could be both fully-stressed and have area equal to the constraint value. In certain problems, especially where a high degree of symmetry is present, this assumption may be violated. The argument presented above for identifying additions or deletions to the optimal fully-stressed set is no longer generally valid. However, the algorithm developed above will still succeed, if the behavior of the specific strain energies is as shown in Fig. 4 Inspection of the non-optimal trial design $D\left(p, S_{1}\right)$ indicates that both members $k$ and $j$ are to be added to the fully-stressed set, since both are stressed above the value $\lambda$. On the other hand, the situation shown in Fig. 5 will lead to failure of the alporithm since iaspection of $D\left(p, S_{1}\right)$ woull indicate (incorrectly) that member $j$ is to be added

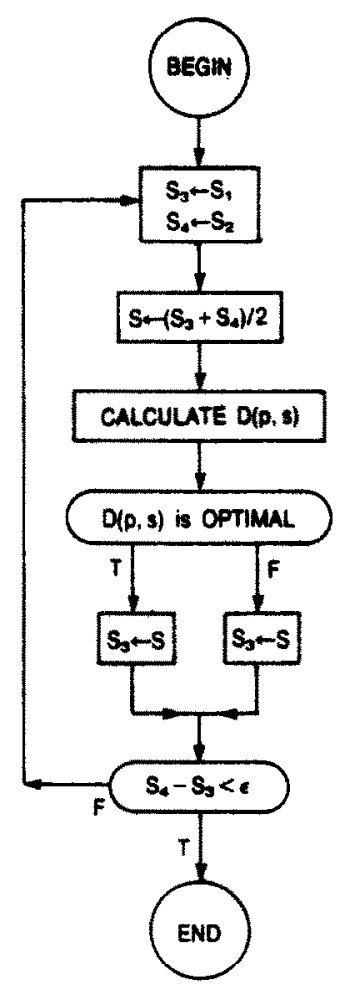

Fig. 2. Algorithm for locating points where optimal fully-stressed set changes. 


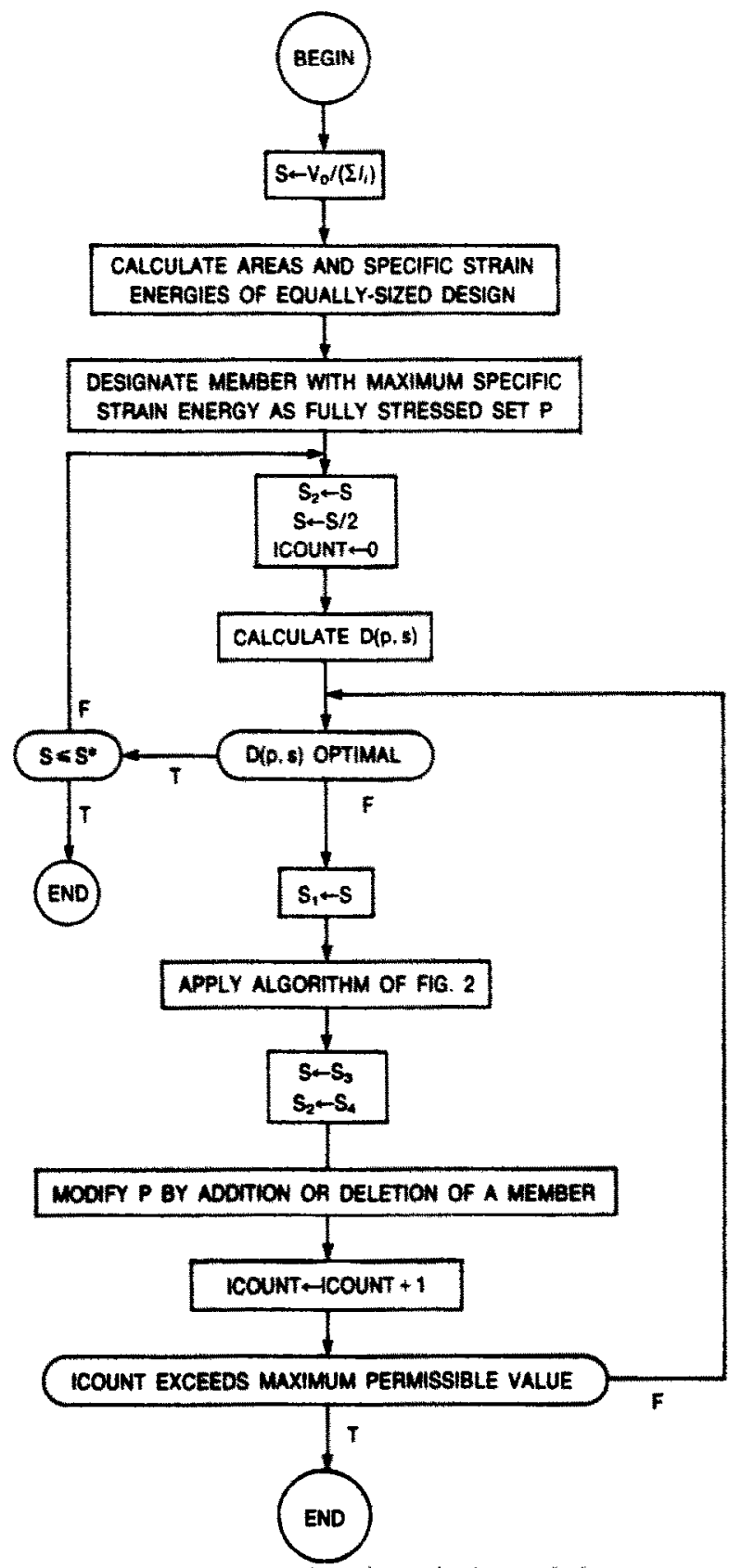

Fig. 3. Agorithm for optimal truss design.

to $p$ to form the optimal fully-stressed set corresponding to $S=S_{1}$. Note that the failure of the algorithm in this instance is caused by the existence of a member which is fully-stressed only for a single value of $S$, rather than over a finite interval.

In the examples considered in the course of this study, several instances were observed where more than one member were fully-stressed and also at the constraint for the same value of S. However, the algorithm had no difficulty in these instances and found the optimal fully-stressed set. The information gained by examining the non-optimal design in the vicinity of a change in the fully-stressed set was a reliable guide in determining the elements to be added or deleted. Thus the lack of theoretical justification for the algorithm in this situation does not appear to be serious. Furthermore failure of the algorithm to produce an optimal design for some value of the lower bound constraint is easily recognized and an appropriate warning produced by the computer program. The troublesome example can then be examined more closely and the members causing the difficulty added or deleted one at a time and in several different orders until an optimal design is found. 


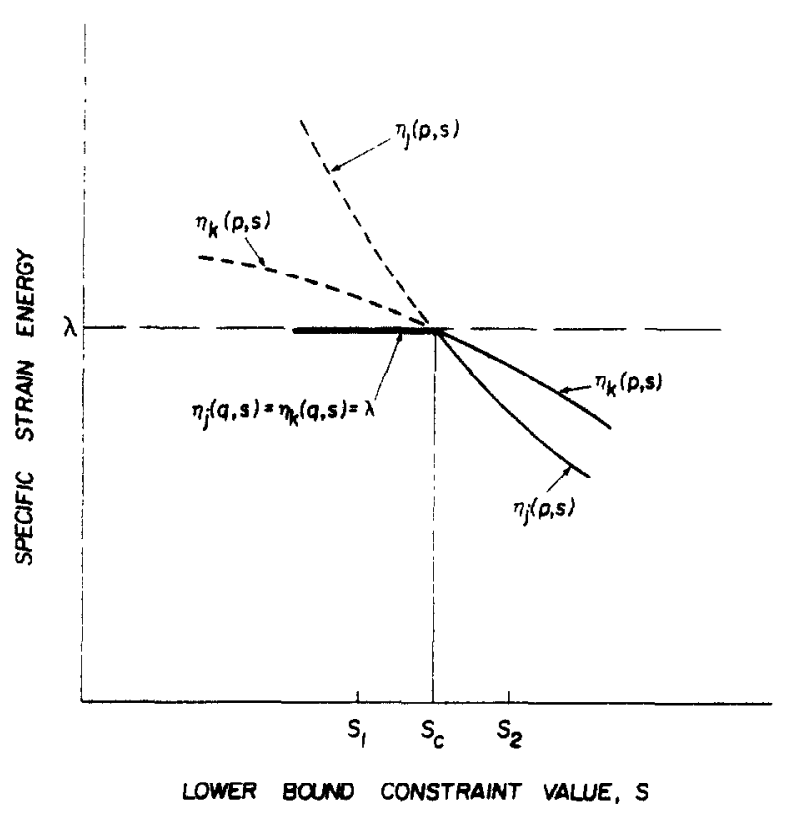

Fig. 4. Members $k$ and $j$ to be added to optimal fully-stressed set.

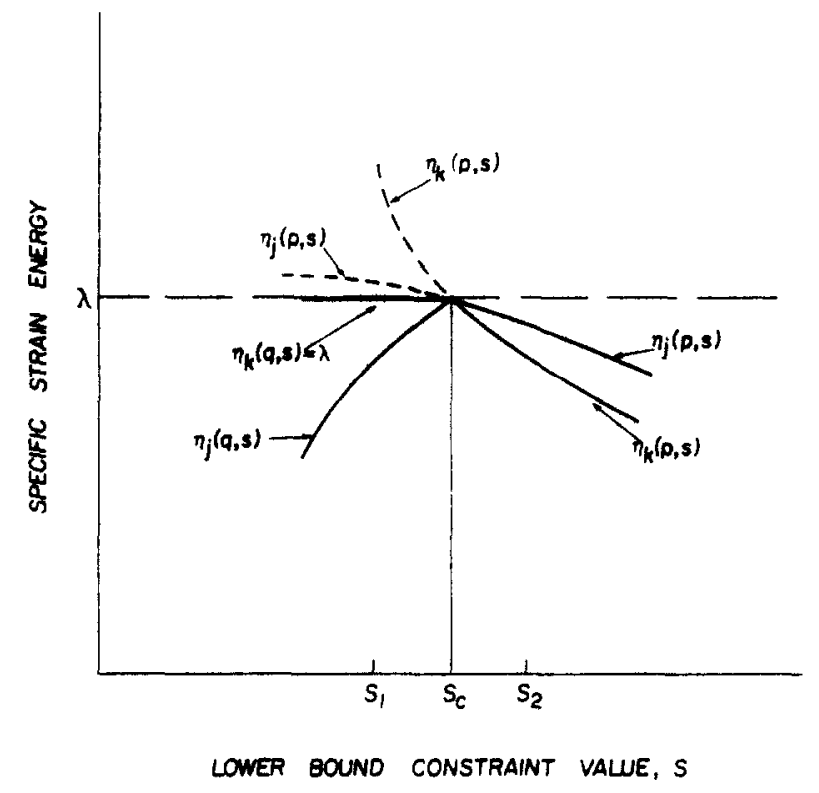

Fig. 5. Member $j$ belongs to optimal fully-stressed set at a single point $\left(S=S_{c}\right)$ only.

\section{EXAMPLE PROBLEMS}

In order to illustrate the results of the preceding paragraphs, several example problems were solved.

The first example, shown in Fig. 6, was chosen to demonstrate the behavior of the optimal design as the lower bound constraint varies, since this is the basis for the algorithm described above. In the figure, twelve support nodes are located along a vertical wall, and the single interior node is loaded with horizontal and vertical forces of nondimensional magnitudes 0.65 and 0.75. All tweive possible truss members are also shown in the figure.

In Fig. 7, the nondimensional areas of the elements of the optimal fully-stressed set are plotted as a function of the lower bound constraint. Note that as the lower bound constraint decreases, the optimal fully-stressed set consists of, successively, member 11 alone, members 11 and 12 , member 12 alone, and finally members 12 and 1 . The optimal design for $S=0$ consists of members 12 and 1 alone, with all other members vanishing. It is interesting that even in this 


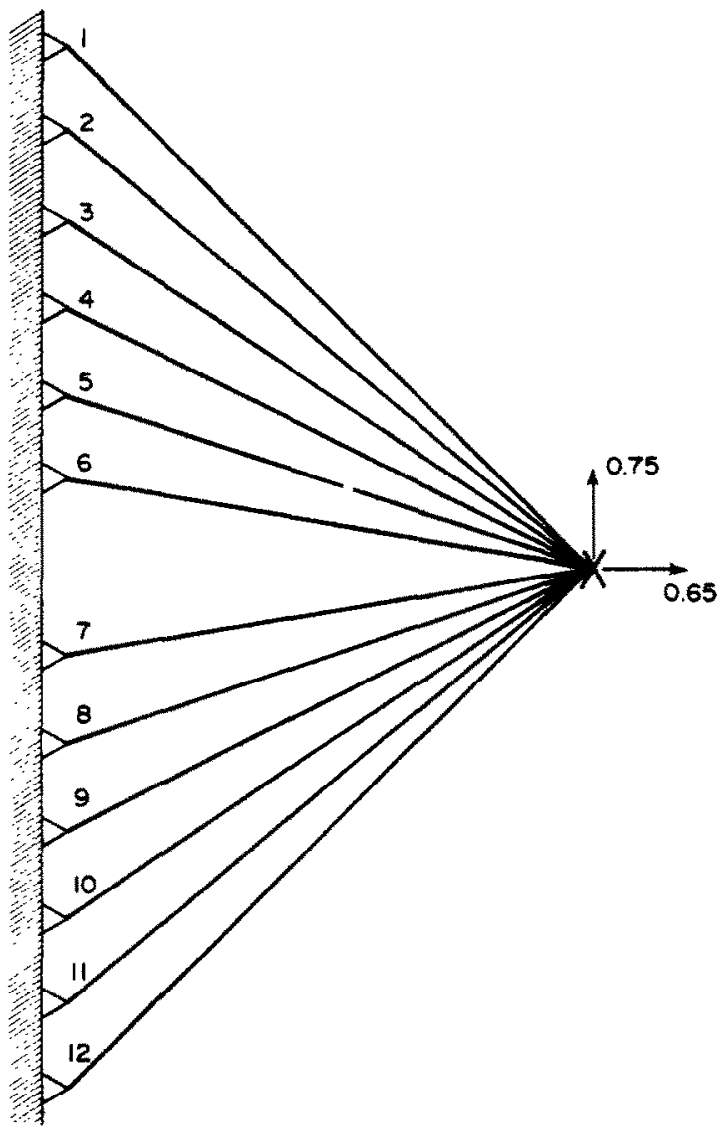

Fig. 6. Truss members to be considered for optimal design problem with one loaded nade and twelve support nodes.

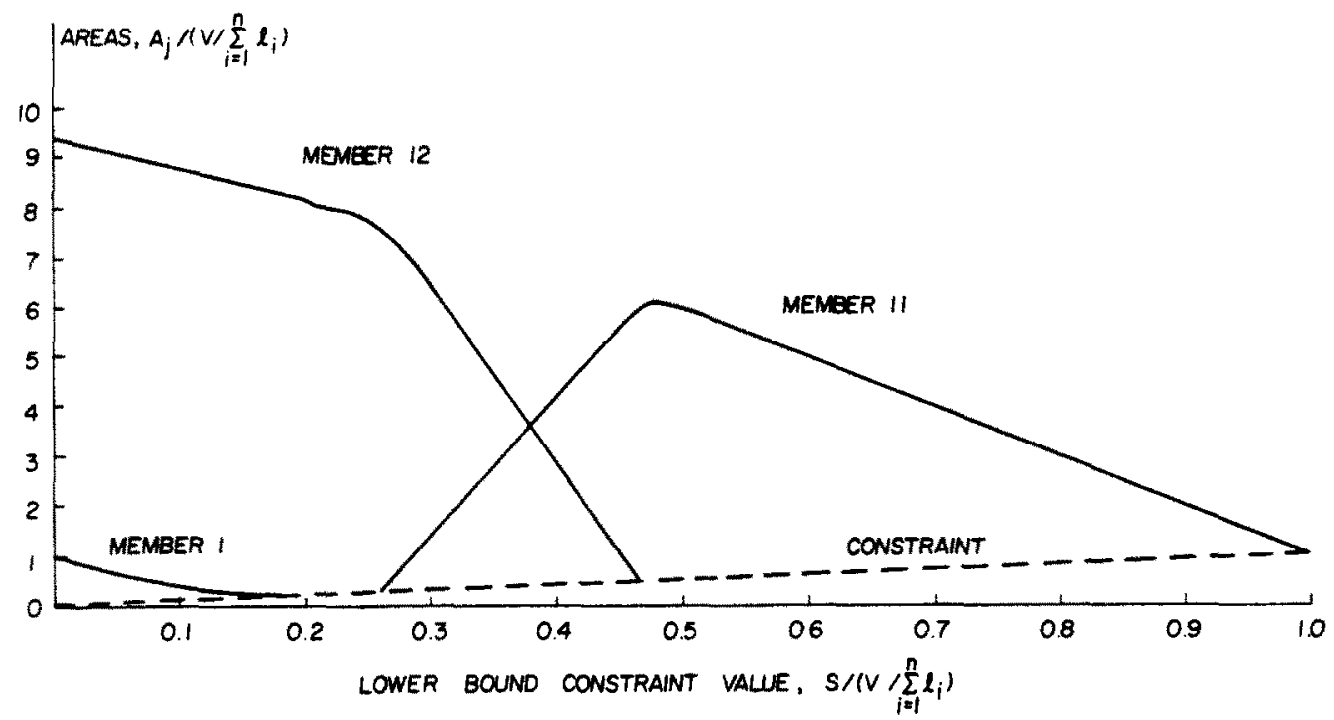

Fig. 7. Areas of fully-stressed elements versus lower bound value.

straightforward example both additions to and a deletion from the optimal fully-stressed set are necessary.

Another example is given in Fig. 8, where a total of eight interior nodes are loaded as indicated by the vectors and accompanying numbers. Two support nodes located far from the interior nodes are not shown in the figure. These supports are not needed in the final result, 


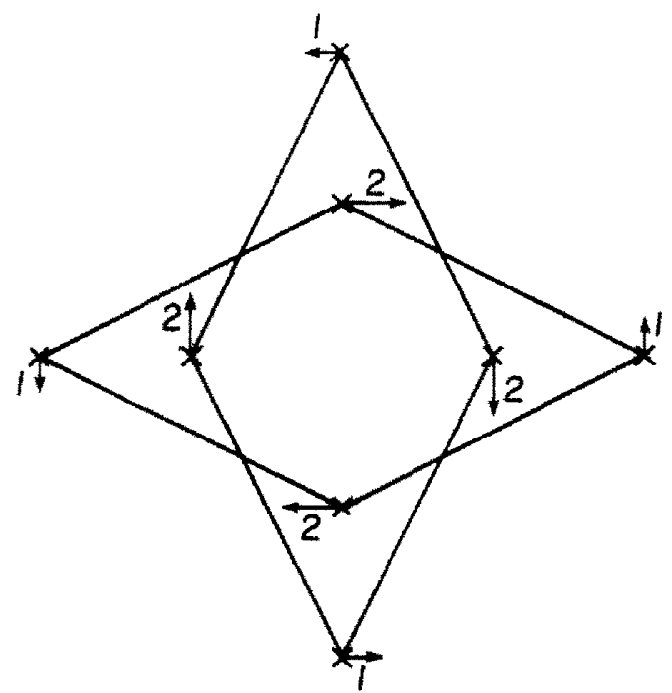

Fig. 8. Self-equilibrated truss, with eight interior nodes.

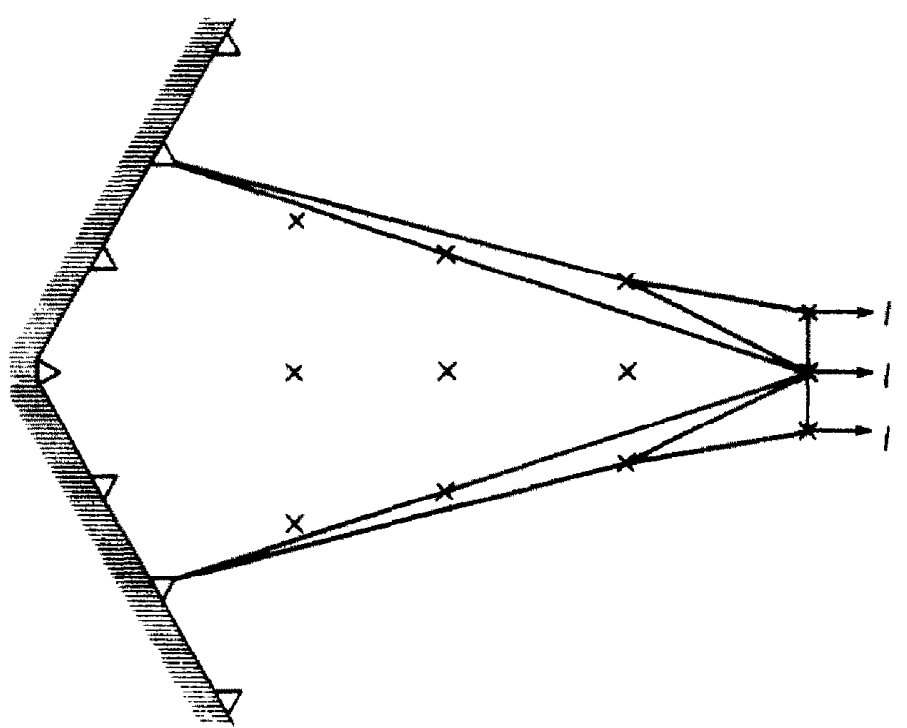

Fig. 9. Optimal truss, with twelve interior nodes and seven support nodes.

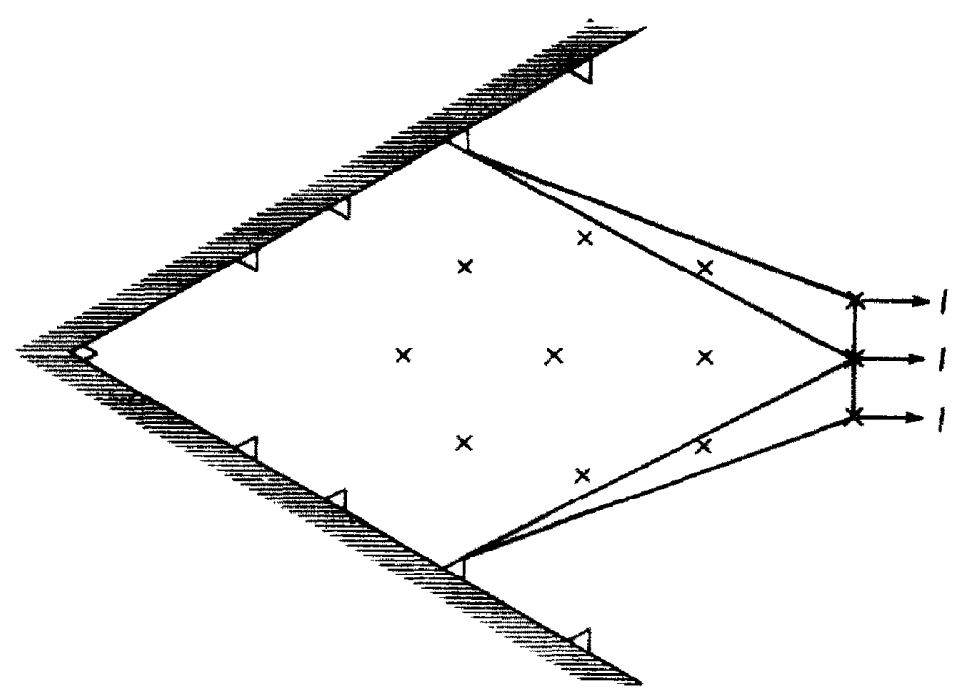

Fig. 10. Optimal truss, with twelve interior nodes and nine support nodes. 
since the optimal design $(S=0)$ found by the algorithm and given in the figure is selfequilibrated. Note that to be practical, the optimal design would require the addition of secondary members to prevent kinematic instability.

Two additional optimal design $(S=0)$ examples are given in Figs. 9 and 10. In both these examples, nine of the twelve interior nodes are unloaded and three loaded with horizontal forces of unit magnitude. Note that the optimal design of Fig. 9 makes use of four unloaded nodes to transmit the applied load to the supports at the wall. However, in Fig. 10 the support nodes are closer to the points of application of the applied loads, and the optimal design transmits the force through members going directly to the supports and ignoring the unloaded nodes.

Finally, in Fig. 11, seven internal and four support nodes are specified, and a single applied load is to be carried by the truss. The optimum design $(S=0)$ is found to contain ten members and is reminiscent of a Michell truss [9].

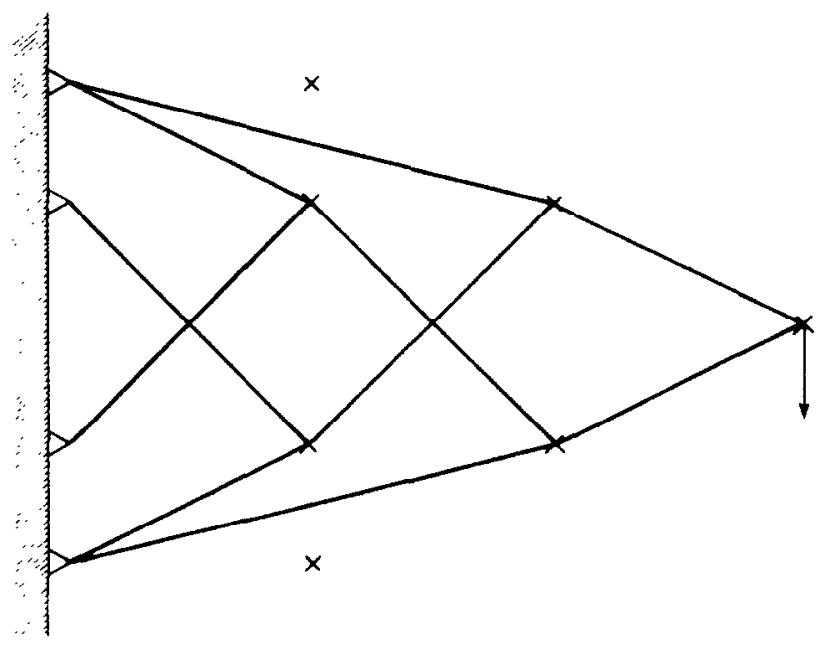

Fig. 11. Optimal truss, with seven interior nodes and four support nodes.

\section{CONCLUSIONS}

As the example problems show, the algorithm can be used successfully to predict optimal truss designs including as a special case optimal layout. Examination of changes in member strain energy as the area constraint is decreased appears to be a reliable guide in deciding which members are to remain fully-stressed and which are not. This examination must, however, be made specifically in the neighborhood of a point where the fully-stressed set changes membership. The primary computational burden of the algorithm stems from attempting to locate such neighborhoods. The development of an extrapolation technique which predicts their location would be a useful extension of the present work.

Acknowledgement-The research reported in this paper was supported under National Science Foundation Grant No. GK-31476.

\section{REFERENCES}

1. W. S. Dorn, R. E. Gomory and H. S. Greenberg, Automatic design of optimal structures. J. de Mécanique. 3, 25-52 1964.

2. J. E. Taylor and M. P. Rossow, An optimal structural design algorithm using optimality criteria. 13th Ann. Meeting Soc. Engineering Sci. Hampton, VA, (3 November 1976).

3. J. E. Taylor, Maximum strength elastic structural design. Proc. ASCE, 95, No. EM3, 653-663 (June 1969).

4. D. J. Hiley, On the optimal design of trusses. M. S. Thesis, University of California at Los Angeles (1968).

5. M. P. Rossow and J. E. Taylor, A finite element method for the optimal design of variable thickness sheets, AIAA J. 11, 1566-1567 (1973).

6. M. P. Rossow, A finite element approach to optimal structural design. Ph.D. Thesis, University of Michigan (1973).

7. Z. Mroz, Multi-parameter optimal design of plates and shells. J. Structural Mech. 1, 371-392 (1973).

8. D. Salinas, On variational formulations for optimal structural design. Ph.D. Thesis, University of California at Los Angeles (1968).

9. A. G. M. Michell, The limits of economy of material in frame-structures. Phil. Mag. 8, 489-497 (1904). 